\title{
Convolutional Neural Networks for Financial Text Regression
}

\author{
Neşat Dereli ${ }^{\dagger}$ and Murat Saraçlar ${ }^{\ddagger}$ \\ ${ }^{\dagger}$ Institute of Graduate Studies in Science and Engineering \\ ${ }^{\ddagger}$ Electrical and Electronics Engineering Department \\ Boğaziçi University, Istanbul, Turkey \\ nesat.dereli@boun.edu.tr, murat.saraclaraboun.edu.tr
}

\begin{abstract}
Forecasting financial volatility of a publiclytraded company from its annual reports has been previously defined as a text regression problem. Recent studies use a manually labeled lexicon to filter the annual reports by keeping sentiment words only. In order to remove the lexicon dependency without decreasing the performance, we replace bag-of-words model word features by word embedding vectors. Using word vectors increases the number of parameters. Considering the increase in number of parameters and excessive lengths of annual reports, a convolutional neural network model is proposed and transfer learning is applied. Experimental results show that the convolutional neural network model provides more accurate volatility predictions than lexicon based models.
\end{abstract}

\section{Introduction}

Most financial analysis methods and portfolio management techniques are based on risk classification and risk prediction. Stock return volatility is a solid indicator of the financial risk of a company. Therefore, forecasting stock return volatility successfully creates an invaluable advantage in financial analysis and portfolio management. While most of the studies are focusing on historical data and financial statements when predicting financial volatility of a company, some studies introduce new fields of information by analyzing soft information which is embedded in textual sources.

Kogan et al. (2009) defined the problem of forecasting financial volatility from annual reports as a text regression task and other studies contributed to the task because of its value (Wang et al., 2013; Tsai and Wang, 2014; Rekabsaz et al., 2017). There are also alternative soft information sources used for financial forecast like news (Tetlock et al., 2008; Nuij et al., 2014; Kazemian et al., 2014;
Ding et al., 2015), online forums (Narayanan et al., 2009; Nguyen and Shirai, 2015), blogs (Bar-Haim et al., 2011) and bank reports (Nopp and Hanbury, 2015). However, annual reports are more informative and contain less noise since they are regulated by the government. On the other hand, annual reports are not suitable for short-term forecasting.

Volatility prediction using annual reports of companies is also a proper test-bed for natural language processing (NLP) since both volatility data and annual report data are freely available and no manual labeling is needed. In U.S., annual report filings, known as $10-\mathrm{K}$ reports, are mandated by the government in a strictly specified format.

Previous works focus on sentiment polarity while forecasting the volatility. Their models are built on top of a financial lexicon (Loughran and McDonald, 2011) and most improvements are obtained by expanding the lexicon. However, a manually created lexicon should be updated over time and the solutions, depending on the lexicon, are not persistent.

In this paper, we propose an artificial neural network (ANN) solution which does not use a lexicon or any other manually labeled source. The convolutional neural network (CNN) model is designed similar to Bitvai and Cohn (2015) and Kim (2014). Nonetheless, annual reports contain excessively long text compared to movie reviews and this results in a more difficult task. To overcome this difficulty, max-over-time pooling layer is replaced by local max-pooling layer and transfer learning is applied.

The rest of the paper is organized as follows. In Section 2, we defined the problem. Section 3 introduces the model and its architecture. The details of our experimental settings, the results of the experiments and the analyses are presented in Section 4. Our work is concluded in Section 5. 


\section{Problem Definition}

In this section, stock return volatility which is aimed to be predicted is defined. Later, the dataset which is used in this work is introduced. Finally, evaluation measures are described.

\subsection{Stock Return Volatility}

Stock return volatility is defined as the standard deviation of adjusted daily closing prices of a target stock over a period of time (Kogan et al., 2009; Tsai and Wang, 2014; Rekabsaz et al., 2017; Hacisalihzade, 2017). Let $S_{t}$ be the adjusted closing stock price for the day $t$. Then, the stock return for the day $t$ is $R_{t}=\frac{S_{t}}{S_{t-1}}-1$. Stock return volatility $v_{[t-\tau, t]}$ for $\tau$ days is given as

$$
v_{[t-\tau, t]}=\sqrt{\sum_{i=0}^{\tau} \frac{\left(R_{t-i}-\bar{R}\right)^{2}}{\tau}} .
$$

\subsection{Dataset}

In this work, the dataset from Tsai et al. (2016), which is published online ${ }^{1}$, is used because it includes up-to-date years and has enough reports for each year. Note that the datasets shared by Kogan et al. (2009) and Rekabsaz et al. (2017) are different than the dataset shared by Tsai et al. (2016) even if the same year is compared since the number of reports differ from each other. Hence, a direct performance comparison is not meaningful.

The dataset from Tsai et al. (2016) includes $10-\mathrm{K}$ reports available on the U.S. Security Exchange Commission (SEC) Electronic Data Gathering, Analysis and Retrieval (EDGAR) website ${ }^{2}$. Following previous works (Kogan et al., 2009; Wang et al., 2013; Tsai and Wang, 2014; Tsai et al., 2016), section 7, Management's Discussion and Analysis (MD\&A) is used instead of the complete $10-\mathrm{K}$ report.

The dataset includes a volatility value for each report of 12 months after the report is published. The volatility value in the dataset is the natural logarithm of stock return volatility and used as the prediction target. We checked randomly sampled reports from SEC EDGAR and calculated volatility values by using adjusted closing stock prices from Yahoo Finance ${ }^{3}$. Both were consistent with the dataset.

\footnotetext{
${ }^{1}$ https://clip.csie.org/10K/data

${ }^{2}$ https://www.sec.gov/edgar.shtml

${ }^{3}$ https://finance.yahoo.com
}

\subsection{Evaluation}

Mean Square Error (MSE) is chosen as the main evaluation metric which is calculated by

$$
M S E=\frac{1}{n} \sum_{i=1}^{n}\left(y_{i}-\hat{y}_{i}\right)^{2}
$$

where $y_{i}=\ln \left(v_{i}\right)$.

Spearman's rank correlation coefficient is a measure which is used to evaluate the ranking performance of a model. Real volatility values and predicted volatility values can be used to calculate Spearman's rank correlation coefficient. Each set contains samples which consist of a company identifier and the volatility value of the company. Spearman's rank correlation coefficient of two sets is equal to Pearson's correlation coefficient of the rankings of the sets. The rankings of a set can be generated by sorting the volatility values of the set in an ascending order and enumerating them. The rankings of a set contains samples which consist of a company identifier and a volatility rank of the company. Spearman's rank correlation coefficient of the sets $X$ and $Y$ can be calculated by

$$
\rho_{X, Y}=\frac{\operatorname{cov}\left(\operatorname{rank}_{X}, \operatorname{rank}_{Y}\right)}{\sigma_{\operatorname{rank}_{X}} \sigma_{\operatorname{rank}_{Y}}}
$$

where $\operatorname{rank}_{X}$ and $\operatorname{rank}_{Y}$ represent the rankings of the sets $X$ and $Y$ respectively.

In all experiments, MSE is used as the loss function which means each model tries to optimize MSE. On the other hand, Spearman's rank correlation coefficient is reported only to evaluate the ranking performance of different models.

\section{Model}

The architecture of the network is presented in Figure 1 which is similar to previous works using CNN for NLP (Collobert et al., 2011; Kim, 2014; Bitvai and Cohn, 2015). Before reports are fed into embedding layer, their lengths are fixed to $m$ words and reports with less than $m$ words are padded. The output matrix of the embedding layer, $E \in \mathbb{R}^{k m}$, consists of $k$-dimensional word vectors where the unknown word vector is initialized randomly and the padding vector is initialized as zero vector. Each element of the word vector represents a feature of the word.

The convolution layer consist of different kernel sizes where each kernel size represents a different $n$-gram. Figure 1 shows tri-gram, four-gram 


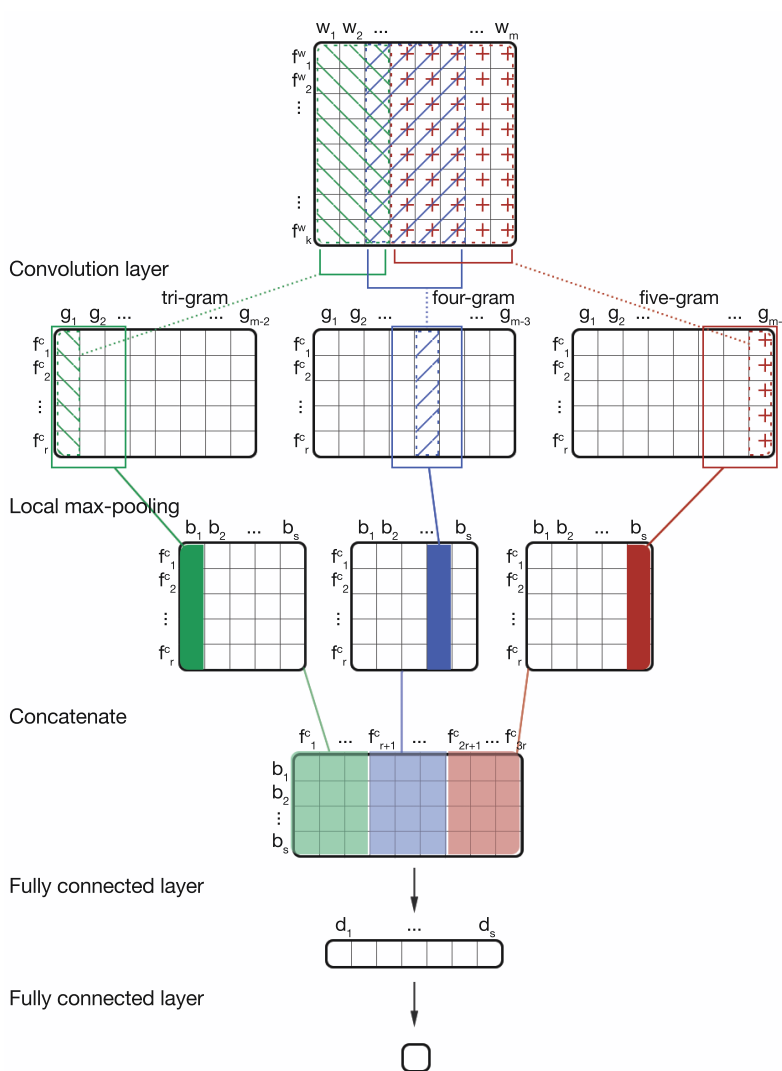

Figure 1: Network architecture of our baseline model (CNN-simple). A word embedded report through a single channel convolution layer with kernel sizes 3, 4 and 5 followed by a local max-pooling and two fully connected layers.

and five-gram examples. Let $n \in \mathbb{N}$ be the kernel width of a target $n$-gram. Each convolution feature $f_{i}^{c} \in \mathbb{R}^{m-n+1}$ is generated from a distinct kernel weight, weight $t_{i}^{n} \in \mathbb{R}^{k n}$, and bias, bias $i \in \mathbb{R}$. Rectified linear unit (ReLU) is used as the nonlinear activation function at the output of the convolution layer,

$$
f_{i j}^{c}=\operatorname{ReLU}\left(\text { weight }_{i}^{n} \cdot w_{j: j+n-1}+\text { bias }_{i}\right) .
$$

Note that the convolution features, $f_{i}^{c}$ have $m-$ $n+1$ dimension and they contain different information than word features, $f_{i}^{w}$. Convolution features are concatenated as

$$
f_{i}^{c}=\left[f_{i 1}^{c}, f_{i 2}^{c}, \ldots, f_{i+n-1}^{c}\right] .
$$

$g_{i}$ in Figure 1 represents each n-gram element thus there are $m-n+1 n$-gram elements for each individual n-gram. Next step is local max-pooling layer which basically applies max-over-time pooling to smaller word sequence instead of the complete text (Le et al., 2018). Each sequence length is $h$ and there are $s$ outputs for each sequence,

$$
b_{i}=\max \left(g_{i h: i(h+1)-1}\right)
$$

where $b_{i} \in \mathbb{R}^{r}$. After the local max-pooling layer is applied to all convolution layer output matrices, they are merged by concatenating feature vectors. Later, dropout is applied to the merged matrix and finally it is fed into two sequential fully connected layers. The presented neural network is implemented by using Pytorch ${ }^{4}$ deep learning framework.

\section{Experiments and Results}

This section states preprocessing operations which are applied to the dataset. Pretrained word embeddings which are used in this work are described. Later, details of the setup of our experiments and the model variations are presented. Finally, the results of the experiments and the analysis of the results are discussed.

\subsection{Preprocessing}

MD\&A section of the $10-\mathrm{K}$ reports in the dataset are already tokenized by removing punctuation, replacing numerical values with \# and downcasing the words. As in previous works, reports are stemmed by using the Porter stemmer (Porter, 1980), supported by Natural Language Toolkit $(\text { NLTK })^{5}$. Stemming decreases the vocabulary size of the word embeddings and thus reduces the parameters of the model. Stemming is also required to use word vectors trained by Tsai et al. (2016) since the corpora which is used to train the word embeddings consists of stemmed reports.

\subsection{Word Embedding}

Word embedding is a method, used to represent words with vectors to embed syntactic and semantic information. Instead of random initialization of the embedding layer of the model, initialization with pretrained word embeddings enables the model to capture contextual information faster and better. In our work, we used pretrained word embeddings supported by Tsai et al. (2016). They used MD\&A section of 10-K reports from 1996 to 2013 to train the word embeddings with a vector dimension of 200 by word $2 \mathrm{vec}^{6}$ continuous bagof-words (CBOW) (Mikolov et al., 2013).

\footnotetext{
${ }^{4}$ https://pytorch.org

${ }^{5}$ https://www.nltk.org

${ }^{6} \mathrm{https}: / /$ code.google.com/archive/p/word2vec/
} 


\begin{tabular}{|l|c|c|c|c|c|c|c|}
\hline Model & 2008 & 2009 & 2010 & 2011 & 2012 & 2013 & Avg \\
\hline EXP-SYN (Tsai 2016) & 0.6537 & $\mathbf{0 . 2 3 8 7}$ & 0.1514 & 0.1217 & 0.2290 & 0.1861 & 0.2634 \\
\hline CNN-simple (baseline) & $\mathbf{0 . 3 7 1 6}$ & 0.4708 & $\mathbf{0 . 1 4 7 1}$ & 0.1312 & 0.2412 & 0.2871 & 0.2748 \\
\hline CNN-STC & 0.5358 & 0.3575 & 0.3001 & 0.1215 & 0.2164 & 0.1497 & 0.2801 \\
\hline CNN-NTC-multichannel & 0.5077 & 0.4353 & 0.1892 & 0.1605 & 0.2116 & 0.1268 & 0.2718 \\
\hline CNN-STC-multichannel & 0.4121 & 0.4040 & 0.2428 & 0.1574 & 0.2082 & 0.1676 & 0.2653 \\
\hline CNN-NTC & 0.4672 & 0.3169 & 0.2156 & $\mathbf{0 . 1 1 5 4}$ & $\mathbf{0 . 1 9 4 4}$ & $\mathbf{0 . 1 2 3 8}$ & $\mathbf{0 . 2 3 8 8}$ \\
\hline
\end{tabular}

Table 1: Performance of different models, measured by Mean Square Error (MSE). Boldface shows the best result among presented models for the corresponding column.

\subsection{Setup}

The hyper-parameters of the CNN models are decided by testing them with our baseline CNN model. All weights of the baseline model are non-static and randomly initialized. Final hyperparameters are selected as mini-batch size 10, fixed text length 20000, convolution layer kernels 3,4 and 5 with 100 output features, probability of dropout layer 0.5, and learning rate 0.001 .

Kogan et al. (2009) showed that using reports of the last two years for training performs better than using reports of the last 5 years. Rekabsaz et al. (2017) presented similarity heat-map of ten consecutive years and stated that groups consist of three to four consecutive years are highly similar. Our experiments also show that including reports which are four years older than test year into training set does not always help and sometimes even causes noise.

In this work, reports of three consecutive years were used for training while reports of the last year were used for validation to determine the best epoch. After the best epoch is determined, it is used as fixed epoch and the oldest year is ignored while the first step is repeated to train a new network without using validation set but fixed epoch instead. For example, reports of 2006 to 2008 are used as training set while reports of 2009 is used for validation. If the best result is achieved after 30 epochs, a new network is trained with reports of 2007 to 2009 through 30 fixed epochs. Finally, the trained network is tested for the year 2010 .

Ignoring years older than four years prevent their noise effect but also reduces training set size. Experiments of this work show that old reports decrease the performance of the embedding layer but increase the performance of the convolution layer. The embedding layer can be biased easier than convolution layer since convolution layer learns features from larger structures (n-grams).
Nonetheless, even training only the convolution layer using all years from 1996 to test year is timeconsuming. Therefore, transfer learning is used by sharing the convolution layer weights which are trained on comparatively larger range of years. Yang et al. (2017) showed that relatedness of the transfer domains has a direct effect on the amount of improvement. Convolution layer weights are trained by freezing the embedding layer which is initialized with pretrained word embeddings and using years 1996 to 2006 for 120 epoch with early stopping. Other hyper-parameters are kept as described above.

\subsection{Extended Models}

Using transfer learning convolution layer, four different models are built. Since convolution layer weights are trained using pretrained word embeddings, those models perform well only when their embedding layers are initialized with pretrained word embeddings. Following Kim (2014), multichannel embedding layers are applied to some models.

- CNN-STC: A model with single channel non-static pretrained embedding layer and a transferred convolution layer which is static.

- CNN-NTC: Same as CNN-STC but its transferred convolution layer is non-static.

- CNN-STC-multichannel: A model with two channel of embedding layers, both are pretrained but one is static and other one is non-static. Transferred convolution layer is also static.

- CNN-NTC-multichannel: Same as CNNSTC-multichannel but its transferred convolution layer is non-static 


\begin{tabular}{|l|c|c|c|c|c|c|c|}
\hline Model & 2008 & 2009 & 2010 & 2011 & 2012 & 2013 & Avg \\
\hline CNN-simple (baseline) & 0.3884 & 0.0814 & $\mathbf{0 . 5 7 5 8}$ & 0.5842 & 0.7064 & 0.7060 & 0.5070 \\
\hline CNN-STC & 0.3875 & $\mathbf{0 . 5 2 2 6}$ & 0.5570 & 0.5737 & $\mathbf{0 . 7 1 4 9}$ & $\mathbf{0 . 7 3 4 1}$ & $\mathbf{0 . 5 8 1 6}$ \\
\hline CNN-NTC-multichannel & 0.3727 & 0.4293 & 0.5187 & 0.5625 & 0.6531 & 0.7332 & 0.5449 \\
\hline CNN-STC-multichannel & 0.3424 & 0.4042 & 0.4641 & 0.4924 & 0.4945 & 0.6305 & 0.4713 \\
\hline CNN-NTC & $\mathbf{0 . 3 9 2 1}$ & 0.4713 & 0.5500 & $\mathbf{0 . 5 9 1 0}$ & 0.6978 & 0.7234 & 0.5709 \\
\hline
\end{tabular}

Table 2: Ranking performance of different models, measured by Spearman's rank correlation coefficient. Boldface shows the best result among presented models for the corresponding column.

\subsection{Results}

Table 1 indicates that performance of our $\mathrm{CNN}$ simple (baseline) model is comparable with EXPSYN, the best model represented by Tsai et al. (2016), which uses a manually created lexicon and POS tagger. Furthermore, the best predictions for the years 2008 and 2010 are achieved by the CNNsimple model. Our best model, CNN-NTC, decreases the average error by $10 \%$ and produces the best predictions for the last three years of the experiment.

Ranking performance is valuable for some real world applications such as portfolio management. Furthermore, better ranking performance indicates better explanation of label distribution. Table 2 shows ranking performance of each model which is presented in this work. Spearman's rank correlation coefficient is bounded between -1 and 1. Higher Spearman's rank correlation coefficient means the model captures larger proportion of variability in the labels. It can be seen that ranking performance of CNN-NTC is as good as its regression performance. On the other hand, $\mathrm{CNN}$ STC can model future distribution of stock return volatilities better than future values of stock return volatilities. It is important to note that our models use MSE as loss function and optimize MSE. Changing the loss function may improve ranking performance results and performance orders of the models.

\subsection{Analysis}

The embedding weights of CNN-NTC are compared with the pretrained word embeddings to determine the most changed words. While comparing the most changed word vectors, the words with yearly frequency less than 250 and more than 5000 are filtered out. Table 3 presents the top 10 most changed words and cosine distances to their pretrained vectors. Note that presented words are stemmed. Since words are in lowercase, the

\begin{tabular}{lc}
\hline Word & Cosine Distance \\
\hline anoth & 0.2565 \\
concern & 0.2436 \\
etc & 0.2431 \\
accordingli & 0.2353 \\
entir & 0.2349 \\
stabil & 0.2328 \\
increment & 0.2308 \\
thu & 0.2306 \\
situat & 0.2167 \\
guaranti & 0.2120
\end{tabular}

Table 3: Top-10 most changed words, extracted from non-static embedding layer.

word ETC may cause confusion. It is an abbreviation and stands for Exchanged-Traded Commodity which is a common word in finance domain and stemmed version includes its plural form ETCs also. The stemmed words concern, stabil and guaranti are sentiment words and contained by finance sentiment lexicon (Loughran and McDonald, 2011). Having 3 sentiment words out of 10 words shows that our model uses sentiment information but not solely depend on sentiment words.

We also analyzed most changed sentiment word, concern, by extracting the 10 nearest words of pretrained word embeddings and CNN-NTC embedding weights separately (Table 4). It can be observed that pertain, about and fear are replaced with safeti, trend and dmaa. Stem words safeti and trend are related with the stem word concern. The word pertain is semantically very close to the word concern, they are even used interchangeably sometimes. However, concern can be replaced with pertain only if it does not have any sentiment polarity. It can be seen that expanding the lexicon using word embeddings, like previous works did (Tsai and Wang, 2014; Tsai et al., 2016; Rekabsaz et al., 2017), can be problematic and may end up with a lexicon expansion contain- 


\begin{tabular}{|c|c|c|c|}
\hline \multicolumn{2}{|c|}{ Static Embedding on 'concern' } & \multicolumn{2}{|c|}{ Non-static Embedding on 'concern' } \\
\hline Word & Cosine Distance & Word & Cosine Distance \\
\hline$\overline{\text { regard }}$ & 0.2772 & $\overline{\text { regard }}$ & 0.3233 \\
\hline privaci & 0.5287 & privaci & 0.5433 \\
\hline inform & 0.5587 & safeti & 0.5550 \\
\hline debat & 0.5706 & inform & 0.5562 \\
\hline implic & 0.5817 & trend & 0.5568 \\
\hline heighten & 0.5825 & heighten & 0.5692 \\
\hline pertain & 0.5844 & inquiri & 0.5959 \\
\hline about & 0.5901 & dmaa & 0.6013 \\
\hline inquiri & 0.5919 & debat & 0.6025 \\
\hline fear & 0.5954 & implic & 0.6033 \\
\hline
\end{tabular}

Table 4: Top-10 most similar words to concern comparing their word vectors.

ing semantically close but sentimentally far words.

Another interesting word in the list is DMAA. It stands for dimethylamylamine which is an energyboosting dietary supplement. In 2012, the U.S. Food and Drug Administration (FDA) warned DMAA manufacturers. In $10-\mathrm{K}$ report of Vitamin Shoppe, Inc. published on February 26, 2013, concern of the company about DMAA is stated:

\begin{abstract}
"If it is determined that DMAA does not comply with applicable regulatory and legislative requirements, we could be required to recall or remove from the market all products containing DMAA and we could become subject to lawsuits related to any alleged non-compliance, any of which recalls, removals or lawsuits could materially and adversely affect our business, financial condition and results of operations."
\end{abstract}

It shows that the CNN model focuses on correct word features but also can overfit easier. In financial text regression task, the word DMAA is quite related with the word concern but it is not a common word and also sector specific.

\section{Conclusion}

The previous studies depend on a financial sentiment lexicon which is initially created by manual work. This paper reduced both dependencies by using word vectors in the model. Word vectors are used in previous studies to expand the lexicon but they are not included to the model directly. On the contrary, our work includes word vectors directly to the model as main input.
In addition, transfer learning is applied to the convolution layer since effect of temporal information on distinct layers differs. Evolving word vectors are analyzed after model benchmarks. The analysis demonstrates that CNN model tracks sentiment polarity of the words successfully and it does not depend on sentiment words only. However, it is also observed that CNN models can overfit easier.

This work is focused on text source and did not include any historical market data or any other metadata. Further research on including metadata to CNN model for the same task may increase the value and analysis.

\section{Acknowledgments}

The numerical calculations reported in this paper were performed at TUBITAK ULAKBIM, High Performance and Grid Computing Center (TRUBA resources). We would like to thank to Can Altay, Çağrı Aslanbaş, Ilgaz Çakın, Ömer Adigüzel, Zeynep Yiyener and the anonymous reviewers for their feedback and suggestions.

\section{References}

Roy Bar-Haim, Elad Dinur, Ronen Feldman, Moshe Fresko, and Guy Goldstein. 2011. Identifying and following expert investors in stock microblogs. In Proceedings of the 2011 Conference on Empirical Methods in Natural Language Processing, pages 1310-1319, Edinburgh, Scotland, UK. Association for Computational Linguistics.

Zsolt Bitvai and Trevor Cohn. 2015. Non-linear text regression with a deep convolutional neural network. In Proceedings of the 53rd Annual Meeting of the Association for Computational Linguistics and the 
7th International Joint Conference on Natural Language Processing (Volume 2: Short Papers), pages 180-185, Beijing, China. Association for Computational Linguistics.

Ronan Collobert, Jason Weston, Léon Bottou, Michael Karlen, Koray Kavukcuoglu, and Pavel Kuksa. 2011. Natural language processing (almost) from scratch. J. Mach. Learn. Res., 12:2493-2537.

Xiao Ding, Yue Zhang, Ting Liu, and Junwen Duan. 2015. Deep learning for event-driven stock prediction. In Proceedings of the 24th International Conference on Artificial Intelligence, IJCAI'15, pages 2327-2333. AAAI Press.

S.S. Hacisalihzade. 2017. Control Engineering and Finance, Lecture Notes in Control and Information Sciences, page 49. Springer International Publishing.

Siavash Kazemian, Shunan Zhao, and Gerald Penn. 2014. Evaluating sentiment analysis evaluation: A case study in securities trading. In Proceedings of the 5th Workshop on Computational Approaches to Subjectivity, Sentiment and Social Media Analysis, pages 119-127, Baltimore, Maryland. Association for Computational Linguistics.

Yoon Kim. 2014. Convolutional neural networks for sentence classification. In Proceedings of the 2014 Conference on Empirical Methods in Natural Language Processing (EMNLP), pages 1746-1751, Doha, Qatar. Association for Computational Linguistics.

Shimon Kogan, Dimitry Levin, Bryan R. Routledge, Jacob S. Sagi, and Noah A. Smith. 2009. Predicting risk from financial reports with regression. In Proceedings of Human Language Technologies: The 2009 Annual Conference of the North American Chapter of the Association for Computational Linguistics, pages 272-280, Boulder, Colorado. Association for Computational Linguistics.

Hoa Le, Christophe Cerisara, and Alexandre Denis. 2018. Do convolutional networks need to be deep for text classification?

Tim Loughran and Bill McDonald. 2011. When is a liability not a liability? textual analysis, dictionaries, and 10-ks. The Journal of Finance, 66(1):35-65.

Tomas Mikolov, Ilya Sutskever, Kai Chen, Greg S Corrado, and Jeff Dean. 2013. Distributed representations of words and phrases and their compositionality. In C. J. C. Burges, L. Bottou, M. Welling, Z. Ghahramani, and K. Q. Weinberger, editors, $A d$ vances in Neural Information Processing Systems 26, pages 3111-3119. Curran Associates, Inc.

Ramanathan Narayanan, Bing Liu, and Alok Choudhary. 2009. Sentiment analysis of conditional sentences. In Proceedings of the 2009 Conference on Empirical Methods in Natural Language Processing, pages 180-189, Singapore. Association for Computational Linguistics.
Thien Hai Nguyen and Kiyoaki Shirai. 2015. Topic modeling based sentiment analysis on social media for stock market prediction. In Proceedings of the 53rd Annual Meeting of the Association for Computational Linguistics and the 7th International Joint Conference on Natural Language Processing (Volume 1: Long Papers), pages 1354-1364, Beijing, China. Association for Computational Linguistics.

Clemens Nopp and Allan Hanbury. 2015. Detecting risks in the banking system by sentiment analysis. In Proceedings of the 2015 Conference on Empirical Methods in Natural Language Processing, pages 591-600, Lisbon, Portugal. Association for Computational Linguistics.

Wijnand Nuij, Viorel Milea, Frederik Hogenboom, Flavius Frasincar, and Uzay Kaymak. 2014. An automated framework for incorporating news into stock trading strategies. IEEE Trans. on Knowl. and Data Eng., 26(4):823-835.

MF Porter. 1980. An algorithm for suffix stripping. Program: Electronic Library and Information Systems, 14.

Navid Rekabsaz, Mihai Lupu, Artem Baklanov, Alexander Dür, Linda Andersson, and Allan Hanbury. 2017. Volatility prediction using financial disclosures sentiments with word embedding-based ir models. In Proceedings of the 55th Annual Meeting of the Association for Computational Linguistics (Volume 1: Long Papers), pages 1712-1721, Vancouver, Canada. Association for Computational Linguistics.

Paul C. Tetlock, Maytal Saar-Tsechansky, and Sofus Macskassy. 2008. More than words: Quantifying language to measure firms' fundamentals. The Journal of Finance, 63(3):1437-1467.

Ming-Feng Tsai and Chuan-Ju Wang. 2014. Financial keyword expansion via continuous word vector representations. In Proceedings of the 2014 Conference on Empirical Methods in Natural Language Processing (EMNLP), pages 1453-1458, Doha, Qatar. Association for Computational Linguistics.

Ming-Feng Tsai, Chuan-Ju Wang, and Po-Chuan Chien. 2016. Discovering finance keywords via continuous-space language models. ACM Trans. Manage. Inf. Syst., 7(3):7:1-7:17.

Chuan-Ju Wang, Ming-Feng Tsai, Tse Liu, and ChinTing Chang. 2013. Financial sentiment analysis for risk prediction. In Proceedings of the Sixth International Joint Conference on Natural Language Processing, pages 802-808, Nagoya, Japan. Asian Federation of Natural Language Processing.

Zhilin Yang, Ruslan Salakhutdinov, and William W. Cohen. 2017. Transfer learning for sequence tagging with hierarchical recurrent networks. 